\title{
Size Dependence of Gold Nanoparticle Interactions with a Supported Lipid Bilayer:
}

\section{A QCM-D Study}

\author{
Christina M. Bailey ${ }^{1}$, Elaheh Kamaloo ${ }^{1}$, Kellie L. Waterman ${ }^{1}$, Kathleen F. Wang ${ }^{1,2}$, Ramanathan \\ Nagarajan $^{2 *}$, and Terri A. Camesano ${ }^{1 *}$ \\ ${ }^{1}$ Department of Chemical Engineering, Worcester Polytechnic Institute, Worcester, MA \\ ${ }^{2}$ Molecular Sciences and Engineering Team, Natick Soldier Research Development and \\ Engineering Center, Natick, MA
}

Keywords: Gold nanoparticles, nanoparticle-lipid bilayer interactions, polymer-induced membrane disruption by nanoparticle, QCM-D study of nanoparticle-bilayer interactions, polymer induced nanoparticle cytotoxicity, phenomenological model of nanoparticle-polymerbilayer interactions

\section{Corresponding Author}

*Ramanathan Nagarajan, Molecular Sciences and Engineering Team, Natick Soldier Research, Development \& Engineering Center, 15 Kansas Street, Natick MA 01760; Phone: 508-233-6445; Fax: 508-233-4469; E-mail: Ramanathan.Nagarajan.Civ@mail.mil

*Terri A. Camesano, Department of Chemical Engineering, Worcester Polytechnic Institute, 100 Institute Rd. Worcester, MA 01609; Phone: 508-831-5380; Fax: 508-831-5853; Email: terric@wpi.edu 


\section{ABSTRACT}

Knowledge of nanoparticle (NP) - membrane interactions is important to advances in nanomedicine as well as for determining the safety of NPs to humans and the ecosystem. This study focuses on a unique mechanism of cytotoxicity, cell membrane destabilization, which is principally dependent on the nanoparticle nature of the material rather than on its molecular properties. We investigated the interactions of 2, 5, 10, and $40 \mathrm{~nm}$ gold NPs with supported lipid bilayer (SLB) of L- $\alpha$-phosphatidylcholine using quartz crystal microbalance with dissipation monitoring (QCM-D). Gold NPs were tested both in the absence of and in the presence of polymethacrylic acid (PMAA), used to simulate the natural organic matter (NOM) in the environment. In the absence of PMAA, for all NP sizes, we observed only small mass losses (1 to $6 \mathrm{ng}$ ) from the membrane. This small lipid removal may be a free energy lowering mechanism to relieve stresses induced by the adsorption of NPs, with the changes too small to affect the membrane integrity. In the presence of PMAA, we observed a net mass increase in the case of smaller NPs. We suggest that the increased adhesion between the NP and the bilayer, promoted by PMAA, causes sufficient NP adsorption on the bilayer to overcompensate for any loss of lipid. The most remarkable observation is the significant mass loss (60 $\mathrm{ng}$ ) for the case of $40 \mathrm{~nm}$ NPs. We attribute this to the lipid bilayer engulfing the NP and leaving the crystal surface. We propose a simple phenomenological model to show that the competition between the particlebilayer adhesion energy, the bilayer bending energy, and the interfacial energy at bilayer defect edges allows the larger NPs which become more adhesive because of the polymer adsorption, to be engulfed by the bilayer and leave the crystal surface, causing large mass loss and membrane disruption. The QCM-D measurements thus offer direct evidence that even if NPs are 
intrinsically not cytotoxic, they can become cytotoxic in the presence of environmental organic matter which modulates the adhesive interactions between the nanoparticle and the membrane. 


\section{INTRODUCTION}

Assessing nanomaterial hazards to humans and environmental organisms has proven to be challenging due to the vast diversity in nanomaterial properties (such as molecular composition, aqueous solubility, water-lipid partition coefficient, nanoparticle size, surface area, shape), the wide variations in the biological targets (such as cell lines, biomarkers) and the conditions (such as the dose levels of the nanoparticles, cell contact times) under which the interactions are examined ${ }^{1}$. Many experimental studies of cytotoxicity of engineered nanomaterials have been reported in the literature ${ }^{2}$, most using mammalian cells, although there is an increasing body of literature related to bacteria. These studies have helped identify a number of mechanisms by which nanomaterials induce toxicity, ${ }^{3}$ including: cell damage by generation of reactive oxygen species (ROS), damage to DNA, damage to the functionality of cellular proteins/enzymes, triggering of inflammation, damage to mitochondrial function, and disruption of cell membrane integrity. All of the abovementioned mechanisms of cytotoxicity, with the exception of cell membrane disruption, have been shown possible from the molecular, atomic or ionic species constituting the NP and without requiring the nanoparticle nature of the material. For example, the ROS generation measured with different metal oxide NPs $\left(\mathrm{Fe}_{2} \mathrm{O}_{3}, \mathrm{Co}_{3} \mathrm{O}_{4}, \mathrm{Mn}_{3} \mathrm{O}_{4}\right)$, and with molecular solutions of the same metal oxides, show that ROS generation occurs readily from molecular solutions ${ }^{4}$ and does not require the material to be present in the nanoparticle form. Similar conclusions can be derived from available experimental data for many of the other cytotoxic mechanisms. In contrast, the integrity of the plasma membrane has been studied in the presence of different kinds of NPs such as metallic silver, semiconductor $\mathrm{CdO}$, and metal oxide $\mathrm{MoO}_{3}$, and compared against the effects of their corresponding salt solutions: silver carbonate, cadmium chloride, and sodium molybdate. ${ }^{5}$ In all cases, the soluble salts did not affect the 
plasma membrane integrity, while the three NPs reduced the membrane integrity allowing leakage of a cytosolic enzyme to occur. This suggests that the mechanism of cytotoxicity in the form of membrane destabilization requires the nanoparticle nature of the material and is not caused by the molecular scale action of the constituent molecules. It follows that if cell membrane disruption is purely due to physical factors such as the size, surface charge density, and polarity of NPs, then it must be a generic mechanism operative in the case of all nanomaterials.

An important complication when studying nanoparticle interactions related to environmental systems arises from the presence of natural organic matter (NOM). Structurally, NOM is extremely complex with a three-dimensional macromolecular architecture and consisting of a diverse group of organic molecules ${ }^{6}$. At present, there is no consensus on the primary binding mechanism responsible for the aggregation of molecules giving rise to NOM. However, the nature of the major functional groups present in NOM has been well characterized, with groups such as carboxyl, hydroxyl, phenolic, alcohol, carbonyl and methoxy, all present. NOM has the ability to modify NP properties by adsorbing to the surface of the particles, which has been found to enhance the stability of aqueous NP dispersions and decrease particle aggregation. ${ }^{7,8}$ Typically, NOM is negatively charged, and it impacts the surface properties of NPs by increasing electrostatic repulsion amongst the NPs. Since NOM is ubiquitously found in natural environments, studies of NP activity against environmental organisms should be conducted in the presence of organic species representing NOM.

In this work we focus on how nanoparticle size affects membrane destabilization, in the absence of and in the presence of organic matter simulating NOM. A model membrane system acting as the analog of biological cells is used to obtain systematic information on the nature of 
NP-membrane interactions. Supported lipid bilayers (SLBs) have become a reliable model system for cell membranes because they exhibit many of the properties of biological membranes, such as lateral fluidity, ability to incorporate proteins, and impermeability to ionic species. ${ }^{9}$ The use of supported lipid bilayer, which mimics the basic membrane structure common to all cellular organisms provides a well-defined model membrane platform for studying NP-cell membrane interactions, mitigating data comparison problems arising from the use of different organisms. To simulate the key characteristics of NOM in the environment, we use a polymer, polymethacrylic acid (PMAA) with the carboxyl functional group, which is an important functional unit of NOM.

The SLBs are amenable to probing by many advanced surface science techniques. We used quartz crystal microbalance with dissipation monitoring (QCM-D) to study the interaction of gold NPs with a supported lipid bilayer membrane. Gold is generally not believed to be cytotoxic, and is under active investigation for use in gene transfer and drug delivery applications. ${ }^{10,11}$ Gold NPs at sizes above $2 \mathrm{~nm}$ do not have catalytic activity. ${ }^{12}$ Therefore, the predominant mechanism by which gold NPs in the 2 to $100 \mathrm{~nm}$ range can cause toxicity is believed to be due only to membrane destabilization. The supported lipid bilayer membrane model is thus ideally suited to explore the mechanistic picture of how gold NPs interact with biological cell membranes, under pristine conditions and simulated environmental conditions.

\section{MATERIALS AND METHODS}

\subsection{Gold nanoparticles}


Spherical gold NPs were purchased from NANOCS (New York, NY) with diameters of 2, 5, 10, and $40 \mathrm{~nm}$ and their manufacturer-specified characteristics are described in the Supplemental Information S1. All NP solutions were diluted with ultrapure water (Milli Q) to $7.14 \times 10^{10}$ particles $/ \mathrm{mL}$ at a neutral $\mathrm{pH}$ of 7 for the QCM-D runs. The corresponding mass concentrations of the NPs in the dispersions are calculated to be $47.6 \mathrm{ng} / \mathrm{mL}, 142.8 \mathrm{ng} / \mathrm{mL}, 1,252.4 \mathrm{ng} / \mathrm{mL}$, and $79,325 \mathrm{ng} / \mathrm{mL}$ respectively, for the $2,5,10$, and $40 \mathrm{~nm}$ particles, using the manufacturer provided mass and number concentrations for the four different particle sizes.

\subsection{Lipids and preparation of vesicles}

L- $\alpha$-phosphatidylcholine (egg, chicken) (PC) with purity $>99 \%$ was purchased from Avanti Polar Lipids, Inc. PC powder (1.0 g) was solubilized in $10 \mathrm{~mL}$ of ethanol to yield $100 \mathrm{mg} / \mathrm{mL}$, and stored at $-20^{\circ} \mathrm{C}$. All other chemicals were purchased from Sigma-Aldrich (St. Louis, MO), unless otherwise stated. The egg phosphatidylcholine (PC) vesicle solution was prepared according to published procedures. ${ }^{13}$ as detailed in the Supplemental Information S2.

\subsection{Formation of supported lipid bilayers}

Supported lipid bilayer was formed on quartz crystals in the Q-sense E4 (Biolin Scientific, Sweden) QCM-D instrument. The protocols for preparing and using the crystals are described in

the Supplementary Information S3. To prepare the supported lipid bilayer ${ }^{14}$, Tris-NaCl buffer was flowed over QCM-D sensors at $0.15 \mathrm{~mL} / \mathrm{min}$. with a peristaltic pump, for $10 \mathrm{~min}$. for all experiments. The lipid vesicle solution was added for $8 \mathrm{~min}$. to form a stable SLB. The crystals were rinsed with Tris- $\mathrm{NaCl}$ buffer for 6 min. to remove any unattached lipids. The frequency change measurements were used to confirm the existence of a stable lipid bilayer for further investigations with NPs. 


\subsection{QCM-D measurements of SLB interactions with gold NPs in water}

Measurements of gold NP-SLB interactions were made in ultrapure water. Since the viscosity and density of the solvent contributes to frequency and dissipation changes, the SLB formed using the buffer solution was first rinsed with the flow of ultrapure water for $8 \mathrm{~min}$. All flows through the QCM-D were kept at the rate of $0.15 \mathrm{~mL} / \mathrm{min}$. After establishing this baseline in ultrapure water, gold NPs in water $\left(7.14 \times 10^{10}\right.$ particles $\left./ \mathrm{mL}\right)$ were allowed to flow for $10 \mathrm{~min}$. NP-membrane interactions occur rapidly followed by steady-state frequency and dissipation changes. Thus, a 10 min. time frame was efficient for measuring our observed interactions and

allowing enough time to ensure complete interaction. Previous studies reported in the literature ${ }^{15}$ such as dye-leakage assays, appeared to require several hours to achieve steady state. However, the interactions observed via QCM-D signals occurred quickly on the surface of the SLB and did not require this long a measurement. Following the flow of the NP solution in water, the SLB was rinsed in ultrapure water, followed by rinse with Tris- $\mathrm{NaCl}$ buffer for $8 \mathrm{~min}$. each. For each NP size, at least 5 replicates were performed.

\subsection{QCM-D measurements of SLB interactions with gold NPs in the presence of PMAA}

To investigate the effect of natural organic matter (NOM), QCM-D experiments were done in the presence of polymethacrylic acid (PMAA), chosen as a NOM simulant from the viewpoint of the important carboxyl functionality. A $0.001 \mathrm{~g} / \mathrm{mL}$ solution of PMAA (Polymer Source, Inc., Quebec, Canada with average molecular weight of $6800 \mathrm{Da}$ and narrow size distribution) was sonicated for $30 \mathrm{~min}$. in an ultrasonic cleaner (Bransonic, Danbury, CT) and stored at $7^{\circ} \mathrm{C}$. PMAA remained at a constant neutral $\mathrm{pH}$ of 7. In the QCM-D experiments, once the stable SLB was formed, the following sequence of flows were allowed to occur: first, the flow of ultrapure 
water as described above, then the flow of $0.001 \mathrm{~g} / \mathrm{mL}$ PMAA for $8 \mathrm{~min}$., followed by the flow of gold NP dispersion $\left(7.14 \times 10^{10} \mathrm{NPs} / \mathrm{mL}\right.$ in $0.001 \mathrm{~g} / \mathrm{mL}$ PMAA) for 8 min., then a 0.001 $\mathrm{g} / \mathrm{mL}$ PMAA rinse at the same flow rate for $8 \mathrm{~min}$., followed by the ultrapure water rinse and finally the buffer rinse. The rinses with ultrapure water before the PMAA flow and after the PMAA flow were done to maintain consistent procedural steps. All four sizes of gold NPs were tested, with at least 5 replicates per NP size.

\subsection{Analysis of QCM-D data}

The QCM-D provides measurements of frequency change and dissipation change. The frequency changes $\Delta f_{\mathrm{n}}$ measured at various overtones $\mathrm{n}(\mathrm{n}=3,5,7,9,11)$ of the natural frequency were normalized by the overtone number $\left(\Delta f=\Delta f_{\mathrm{n}} / \mathrm{n}\right)$. For the analysis, $\Delta f$ data for the $3^{\text {rd }}$ to $11^{\text {th }}$ overtones were measured and used. Only small changes occur between different overtones, and the $3^{\text {rd }}$ and $11^{\text {th }}$ overtones were chosen to provide a representation of the highest and lowest resonances. The frequency change at the fundamental frequency is not generally analyzed since this is affected by the flow of bulk solution ${ }^{16}$. The QCM-D also measured energy dissipation changes, $\Delta D$, which provided information on the rigidity of the adsorbed film. Typical sensitivities of QCM-D measurements in liquid are $\sim 0.1 \mathrm{~Hz}$ for frequency (equivalent to an areal mass of $\sim 1.7 \mathrm{ng} / \mathrm{cm}^{2}$ ) and $\sim 0.1 \times 10^{-6}$ for dissipation.

Methods to relate the measured frequency and dissipation changes to changes in mass and in the viscoelastic properties of the membrane on the surface have been described in detail in the literature ${ }^{17-19}$ and only a brief summary is provided here. For a rigid film of areal mass $m_{f}$ (mass per unit area) deposited on the crystal surface and exposed to air, the frequency change $\Delta f$ which is normalized with respect to the overtone number and the areal mass of the film are related by the Sauerbrey equation, while the dissipation change $\Delta D$ is zero. 


$$
\frac{\Delta f_{n}}{n}=\Delta f=-\frac{f_{n}}{n} \frac{m_{f}}{m_{q}}=-f_{o} \frac{m_{f}}{m_{q}}, \Delta D=0
$$

Here, $f_{n}$ is the natural frequency of the oscillator at the overtone number $n, f_{o}$ is the fundamental frequency of the oscillator $(5 \mathrm{MHz})$ and $m_{q}$ is the areal mass of the quartz crystal $\left(0.883 \mathrm{~kg} / \mathrm{m}^{2}\right)$. The mass addition due to the film deposited on the crystal surface gives rise to a decrease in the frequency (negative $\Delta f$ ) while net mass loss is indicated by a positive $\Delta f$. The dissipation $D$ is related to the loss modulus $G^{\prime \prime}$ and the storage modulus $G^{\prime}$ in the form $D=G^{\prime \prime} /\left(2 \pi G^{\prime}\right)$ and the change in dissipation $\Delta D$ can be related to the changes in the rigidity or viscoelasticity of the film attached to the crystal surface. Obviously, for the rigid film, the change in dissipation is zero. The change in mass for a rigid film can be calculated through the Sauerbrey relationship as, $\Delta \mathrm{m}=-\mathrm{C} \cdot \Delta f$, where $\Delta m$ is the change in areal mass (mass per crystal area, measured in $\mathrm{ng} / \mathrm{cm}^{2}$ ) corresponding to the normalized frequency change $\Delta f$ (in units of $\mathrm{Hz}$ ) and $C$ the mass sensitivity constant for the sensor crystal $\left(=17.8 \mathrm{ng} \mathrm{cm}^{-2} \mathrm{~Hz}^{-1}\right.$ for crystals oscillating at natural frequency of $5 \mathrm{MHz}$ ). The areal mass change can be multiplied by the crystal surface area (calculated to be $1 \mathrm{~cm}^{2}$ ) to obtain mass change for the entire crystal.

If the rigid film is immersed in a Newtonian liquid (water or any of the solutions used in our experiments), the frequency and dissipation changes are modified due to the presence of the liquid and are now given by:

$$
\Delta f=-\frac{1}{n} \frac{\eta_{L}}{2 \pi \delta_{L} m_{q}}-f_{o} \frac{m_{f}}{m_{q}}, \Delta D=\frac{\eta_{L}}{n \pi f_{o} \delta_{L} m_{q}}, \quad \frac{\eta_{L}}{\delta_{L}}=\left(n \pi f_{o} \eta_{L} \rho_{L}\right)^{1 / 2}
$$

where $\eta_{L}$ is the viscosity of the liquid medium and $\delta_{L}$ is the decay length of the acoustic wave in the liquid medium. The first term in $\Delta f$ and the term appearing in $\Delta D$ represent the contributions coming from the removal of the quartz crystal from air and immersion in a liquid medium and are influenced by the viscosity and density of the solvent. Usually when measurements of any 
mass changes on the crystal are made using the same liquid, this solvent effect vanishes, since the crystal is in the same liquid both before and after the mass change process. Effectively, the film mass changes are given just by the Sauerbrey term.

If the film is not rigid but viscoelastic, then the frequency and dissipation changes are given by

$$
\begin{gathered}
\Delta f=-\frac{1}{n} \frac{\eta_{L}}{2 \pi \delta_{L} m_{q}}-f_{o} \frac{m_{f}}{m_{q}}\left[1-\frac{2}{\rho_{f}}\left(\frac{\eta_{L}}{\delta_{L}}\right)^{2} \frac{G^{\prime \prime}}{G^{\prime 2}+G^{\prime 2}}\right], \\
\Delta D=\frac{\eta_{L}}{n \pi f_{o} \delta_{L} m_{q}}+\frac{m_{f}}{m_{q}}\left[\frac{4}{\rho_{f}}\left(\frac{\eta_{L}}{\delta_{L}}\right)^{2} \frac{G^{\prime}}{G^{\prime 2}+G^{\prime 2}}\right]
\end{gathered}
$$

As in eq. (2), the first term in the expressions for $\Delta f$ and $\Delta D$ are due to the solvent effect and they vanish when we consider changes in film properties when the film is immersed in the same liquid both before and after the change. The film mass change is now given by the Sauerbrey term with a correction factor accounting for the viscoelastic properties of the film. Further, there are dissipation changes due to the viscoelastic nature of the film.

\section{RESULTS}

\subsection{Interactions of gold NPs in water with the SLB}

The QCM-D response of frequency and dissipation changes in the experiments involving the citric acid-stabilized gold NPs in water are shown in Fig. 1A for $10 \mathrm{~nm}$ NPs and in Fig. 1B for 40 $n m$ NPs. In these figures, the sequence of events ' $a$ ' through ' $\mathrm{f}$ ' represents the various liquid flow processes over the quartz crystal. In stage (a) a stable lipid bilayer was formed and the bilayer formation was monitored via QCM-D in several steps. As the initial Tris-NaCl buffer on the crystal was replaced by the flow of vesicle solution in the same buffer, the vesicles adsorb on the crystal. This was revealed by the initial large decrease in frequency, or increase in mass, and 
a sharp increase in dissipation denoting a soft film formation. The vesicles then rupture spontaneously releasing encapsulated aqueous phase and forming the planar supported bilayer. This was indicated by the large increase in frequency, or decrease in mass, associated with the loss of liquid from the vesicle interior and a significant decrease in dissipation denoting that the soft vesicle layer with the liquid encapsulated inside vesicles and trapped between vesicles was being replaced by a more rigid lipid bilayer film. The membrane was then stabilized during buffer flow in stage (b), which removed any un-ruptured vesicles or not strongly adsorbed lipid fragments. In stage (c) the bilayer was conditioned with the flow of ultrapure water in order to prepare for the nanoparticle contact. The addition of water caused the frequency to increase and dissipation to decrease due to the slightly lower viscosity and density of water that replaced the higher viscosity and density buffer solution. A dispersion of gold NPs in water was then administered to the bilayer and allowed to interact for ten minutes in stage (d). Water was then flowed through the system in stage (e), followed by a final buffer rinse in stage (f). These final rinses allowed for bilayer comparison before and after NP administration.

The changes in $\Delta f$ and $\Delta D$ observed from the end of stage (c) to the end of stage (d) represent the consequences of NP interactions with the lipid bilayer. Eq.(2) demonstrates how the density and viscosity of the solvent affected the frequency and dissipation changes. If the density and viscosity values of the solvent in stage (c) are different from those of the solvent in stage (d), the observed $\Delta f$ and $\Delta D$ values will have contributions arising from the change in the bulk properties of the solvent. We can evaluate whether this is the case in order to decide whether any solvent corrections are needed.

The number of NPs added was constant for all four NP sizes. The largest volume or mass fraction of NPs added corresponds to the $40 \mathrm{~nm} \mathrm{NP}$ and therefore it is expected that the density 
and viscosity changes will be the largest for this system. We calculated the mass concentration of the $40 \mathrm{~nm} \mathrm{NP}$ in the solvent to be $79,325 \mathrm{ng} / \mathrm{mL}$ based on the number concentration of the particles and the mass density of gold. Taking the density of water to be $1 \mathrm{~g} / \mathrm{mL}$, the density of NP-containing solvent (i.e., water + NPs) was $1.0000793 \mathrm{~g} / \mathrm{mL}$, which was not significantly different from that of the NP-free solvent (water). For the three smaller sized NPs, the density of the NP-containing solvent will be even smaller and virtually the same as the density of NP-free solvent (water). Noting that the density of gold is $19.3 \mathrm{~g} / \mathrm{mL}$, the volume fraction of the NPs in the solvent, $\varphi_{\mathrm{NP}}$, was $\sim 0.0000793 / 19.3 \sim 4 \times 10^{-6}$. The ratio between the viscosity of the NPcontaining solvent $\left(\eta_{\mathrm{w}+\mathrm{NP}}\right)$ and the NP-free solvent $\left(\eta_{\mathrm{w}}\right)$ as a function of the volume fraction $\varphi_{\mathrm{NP}}$ of spherical NPs was calculated from the Einstein equation ${ }^{20,21}$ :

$$
\frac{\eta_{w+N P}}{\eta_{w}}=1+2.5 \varphi_{N P}
$$

Since the particle volume fraction $\varphi_{\mathrm{NP}}$ is small, $\sim 4 \times 10^{-6}$, the viscosity of the NP containing solvent will be practically identical to the viscosity of the NP-free solvent. This conclusion is also valid for the other three smaller sized NPs examined in this work, since their volume fractions will be even smaller than that for the $40 \mathrm{~nm}$ NP. Since both the density and the viscosity of NP-containing solvent and NP-free solvent are practically the same, the measured $\Delta f$ and $\Delta D$ values will have no contributions from the solvent bulk properties when we replace the solvent in stage (c) by the solvent in stage (d). Therefore, all observed changes in $\Delta f$ and $\Delta D$ can be confidently assigned solely to the interactions of the NPs with the bilayer.

The observed dissipation changes for all four sizes of NPs at various overtone numbers are negligible (Fig. 2). This implies that the lipid bilayer on the quartz surface can be treated as a rigid film and the frequency changes observed can be directly connected to mass changes through the Sauerbrey relationship eq.(2). From the frequency change measured as the difference 
in frequency at the end of stage (c) and stage (d), areal mass changes were determined for each NP size and at each overtone. Using this areal mass change and taking the crystal active area to be $1 \mathrm{~cm}^{2}$, the total mass changes on the crystal at various overtones were calculated (Fig.3). The calculated mass change values at all overtone numbers were small but negative, indicating that a small mass was lost from the system for all sizes of the NPs, implying the removal of some lipid molecules (Fig.3). The mass loss is in the 1 to 6 ng range indicating that at best 1 or $2 \%$ of the lipids in the bilayer would have to be removed to explain the observed QCM-D response.

\subsection{Interaction of gold NPs with the SLB in the presence of PMAA}

We investigated the influence of organic molecules simulating the presence of NOM in the environment, selecting polymethacrylic acid (PMAA) with carboxyl functional groups. The QCM-D response of frequency and dissipation changes in the experiments involving the NPs in PMAA + water are shown in Fig.1C and Fig.1D for the $10 \mathrm{~nm}$ and $40 \mathrm{~nm}$ NPs, respectively. As in the case of citric acid-stabilized gold NPs in water, the sequence of events 'a' through ' $h$ ' in Fig.1C and Fig.1D represent the various liquid flow processes over the quartz crystal, with the first three stages ( $a, b$, and c) being identical to those discussed. In stage (d) a solution of 0.001 wt $\%$ PMAA in water was allowed to flow over the bilayer. Gold NPs equilibrated in the PMAA solution (0.001 wt $\%$ PMAA in water) were then allowed to interact with the SLB in stage (e). A rinse in the PMAA solution followed in stage (f), followed by a water rinse in stage (g), and a

final buffer rinse in stage (h). The water rinses in stages (c) and (g) were performed to maintain the same sequence of steps as for the experiment with NPs in water.

The mass concentration of PMAA used in the experiments was $10^{-5} \mathrm{~g} / \mathrm{mL}$ and the mass concentration of the $40 \mathrm{~nm} \mathrm{NP}$ in the solvent was $79,325 \mathrm{ng} / \mathrm{mL}$. As the molecular weight of PMAA is $6.8 \mathrm{kDa}$, we do not expect to see a large effect on solution properties from the presence 
of the polymer. Given these values, using the same quantitative arguments discussed in section 3.1, we can conclude that the viscosities of PMAA + water + NPs and PMAA + water were practically identical to the viscosity of water; and the densities of PMAA + water + NPs and PMAA + water were practically identical to the density of water. Therefore, all frequency and dissipation changes monitored were free of any contributions due to solvent bulk property changes.

The changes in $\Delta f$ and $\Delta D$ observed from the end of stage (d) to the end of stage (e) represent solely the consequences of NP interactions with the lipid bilayer in the presence of PMAA. The addition of PMAA to the system caused a size-dependent mechanism for the NP-bilayer interaction (Fig.3). The $2 \mathrm{~nm}$ and $5 \mathrm{~nm}$ gold NPs contributed to a small mass increase on the bilayer surface, indicating NP adsorption on the bilayer while the $10 \mathrm{~nm}$ NPs exhibited a small mass decrease on the surface, which indicated some lipid removal. All of these changes were relatively quite small. The most prominent change was seen with the $40 \mathrm{~nm}$ gold NPs, which caused a large mass decrease on the surface, causing approximately $22 \%$ of the bilayer to be

removed. The frequency changes for each overtone were fairly similar, indicating that the lipid loss was a homogenous process along the depth of the bilayer. The dissipation change for the 40 nm NP was still quite small and negative, implying that the rigidity of the bilayer was maintained even if there was a significant lipid loss from the bilayer (Fig.2).

\subsection{Interaction of PMAA with the SLB before and after NP exposure}

The changes in $\Delta f$ and $\Delta D$ observed from the end of stage (c) to the end of stage (d) in Fig.1C and Fig.1D represent the interactions of PMAA with the lipid bilayer, before the bilayer was exposed to NPs. For the $10 \mathrm{~nm}$ and $40 \mathrm{~nm}$ particles, these $\Delta f$ and $\Delta D$ values are plotted in Fig.4A and Fig.4B, respectively. PMAA's interaction was unaffected by NP size, since all of this 
interaction occurred before the exposure of the bilayer to the NPs. The significant decrease in the frequency and small increase in the dissipation implied that there was significant adsorption of PMAA on to the lipid bilayer, yet the basic structural organization and rigidity of the bilayer had not been affected.

The changes in $\Delta f$ and $\Delta D$ observed from the end of stage (e) to the end of stage (f) in Fig.1C and Fig.1D represent the interactions of PMAA with the lipid bilayer, after the bilayer was exposed to NPs. For the $10 \mathrm{~nm}$ and $40 \mathrm{~nm}$ NPs, these $\Delta f$ and $\Delta D$ values are plotted in Fig. $4 \mathrm{C}$ and Fig.4D, respectively, and size affected these interactions. For the $10 \mathrm{~nm}$ NPs, there were no noticeable changes in frequency or dissipation and the bilayer was not affected by the exposure to the NPs (as discussed in Section 3.2). Further, subsequent contact with the PMAA did not result in any modifications to the bilayer. In contrast, for the $40 \mathrm{~nm} \mathrm{NPs}$, there was a significant frequency decrease, implying that mass was added to the bilayer. Exposure of the bilayer to the NPs caused appreciable mass loss in stage (d) to (e), but this mass loss was more than compensated by a mass increase in stage (e) to (f), implying that the PMAA adsorbed onto the bilayer and adsorbed to defect sites caused by lipid removal.

\section{DISCUSSION}

\subsection{Measured $\Delta f$ and $\Delta D$ represent NP-bilayer interactions and not solvent effects}

A fundamental question in QCM-D experiments is whether any of the measured frequency and dissipation changes could have originated from changes in the bulk properties of solvents. Significant changes in frequency and dissipation are possible even from what may be seen as small changes in solvent density and viscosity, according to the solvent dependent terms in eq.(2). In the present study, the question translates to the differences in the viscosities and 
densities of the solvent compared to the solvent containing NPs. In one case the solvent is water and in the other case it is a solution of PMAA in water. The larger NPs would make the most contributions to density and viscosity. Taking the most extreme case of the $40 \mathrm{~nm}$ NPs, viscosity $(\eta)$ and density $(\rho)$ changes due to the addition of NPs in the NP-free solvent can be written as

$$
\begin{gathered}
\frac{\eta_{w+N P}}{\eta_{w}}=\frac{\eta_{w+P M C+N P}}{\eta_{w+P M C}}=1.000004 \\
\text { and } \\
\frac{\rho_{w+N P}}{\rho_{w}}=\frac{\rho_{w+P M C+N P}}{\rho_{w+P M C}}=1.0000793
\end{gathered}
$$

Corresponding to these bulk property changes, the changes in frequency and dissipation calculated from the solvent-dependent terms in eq.(2) are indeed entirely negligible and therefore, we can conclude that all changes we measured are solely due to the interaction of NPs with the bilayer membrane.

\subsection{Estimating citric acid-stabilized gold NPs adsorption on bilayer}

For the citric acid-stabilized gold NPs, we observed small mass losses for all four sizes of NPs. Mass increases were never observed, even though one might expect possible NP adsorption on the bilayer. We explored this by estimating the extent of NP adsorption on the bilayer by applying results reported by Hou et al., ${ }^{22}$ who studied the partitioning of tannic acidstabilized gold NPs (size 5 to $100 \mathrm{~nm}$ ) between the aqueous phase and the lipid bilayer. They employed commercially available solid-supported lipid membranes (SSLMs), which are silica spheres with a non-covalent coating of egg PC lipid bilayers, and measured the lipid bilayerwater distribution coefficient $\mathrm{K}_{\text {lipw }}\left(=\mathrm{C}_{\text {lip }} / \mathrm{C}_{\mathrm{w}}\right)$ to be $450 \mathrm{~L} / \mathrm{kg}$ lipid. Here $\mathrm{C}_{\text {lip }}$ refers to the concentration of NPs in the lipid domain expressed as mass (or number) of lipid particles per $\mathrm{kg}$ 
of lipid and $\mathrm{C}_{\mathrm{w}}$ is the concentration of NPs in water, expressed as mass (or number) of NPs per liter of the aqueous phase.

In our QCM-D experiments, we used a particle concentration of $\mathrm{C}_{\mathrm{w}}=7.14 \times 10^{13}$ particles $/ \mathrm{L}$, for all sizes. Using the lipid bilayer-water distribution coefficient reported above, we found that the number concentration of NPs in the lipid bilayer would be $\mathrm{C}_{\text {lip }}=3.213 \times 10^{16} \mathrm{particles} / \mathrm{kg}$ of lipid. To calculate the number of NPs adsorbed on the bilayer, we have to determine the mass of lipid on the bilayer. The formation of the supported egg PC bilayer results in a frequency change of $\sim 25 \mathrm{~Hz}$ which corresponds to a bilayer areal mass of $445 \mathrm{ng} / \mathrm{cm}^{2}$, based on the Sauerbrey constant $\left(\mathrm{C}=17.8 \mathrm{ng} \mathrm{cm}^{-2} \mathrm{~Hz}^{-1}\right)$. This mass includes the mass of a layer of water between the quartz crystal and the supported lipid bilayer, which is $\sim 102 \mathrm{ng} / \mathrm{cm}^{223}$. Correcting for this water mass, the areal mass of the lipid is $343 \mathrm{ng} / \mathrm{cm}^{2}$. Combining this with the estimate for $\mathrm{C}_{\text {lip }}$, we find that the number of NPs associated with the bilayer is $1.1 \times 10^{7}$ particles $/ \mathrm{cm}^{2}$. Taking the mass of each NP using data provided by the manufacturer, we calculated the total mass of NPs adsorbed on the bilayer per unit area and the corresponding frequency change on the quartz crystal (Table 1). All estimated frequency changes are negative since they correspond to mass addition resulting from the adsorption of NPs. The results clearly show that for the number density of nanoparticles used in the experiment, the adsorption of NPs on the lipid bilayer would not be detectable by the QCM-D for the 2, 5 and $10 \mathrm{~nm}$ particles and is very small but detectable, for the $40 \mathrm{~nm}$ particle.

Table 1. Estimation of frequency changes due to nanoparticle adsorption on bilayer

\begin{tabular}{|c|c|c|c|}
\hline $\begin{array}{c}\text { NP diameter } \\
(\mathrm{nm})\end{array}$ & $\begin{array}{c}\text { Mass of one NP based } \\
\text { on manufacturer data } \\
(\mathrm{ng})\end{array}$ & $\begin{array}{c}\text { Predicted areal mass of } \\
\text { adsorbed NP }\left(\mathrm{ng} / \mathrm{cm}^{2}\right)\end{array}$ & $\begin{array}{c}\text { Expected frequency change due } \\
\text { to NP adsorption }(\mathrm{Hz})\end{array}$ \\
\hline 2 & $6.67 \times 10^{-10}$ & $7.34 \times 10^{-3}$ & $-0.41 \times 10^{-3}$ \\
\hline
\end{tabular}




\begin{tabular}{|c|c|c|c|}
\hline 5 & $2.0 \times 10^{-9}$ & $2.20 \times 10^{-2}$ & $-0.12 \times 10^{-2}$ \\
\hline 10 & $1.75 \times 10^{-8}$ & $1.93 \times 10^{-1}$ & $-0.11 \times 10^{-1}$ \\
\hline 40 & $11.11 \times 10^{-7}$ & 12.22 & -0.69 \\
\hline
\end{tabular}

\subsection{Citric acid stabilized gold NP adsorption on bilayer causes some lipid removal}

The experimental QCM-D data for all four sizes of citric acid-stabilized gold NPs showed positive frequency changes, corresponding to net mass loss. The mass loss is quite small, in the range 0 to $6 \mathrm{ng}$, and must come from the removal of some lipid molecules from the bilayer. We propose that the NPs adsorb on the surface and the NP adsorption causes displacement of lipid head groups due to the surface insertion of the particle (Fig.5). This affects the lipid conformation and the resulting membrane stresses are relieved by the escape of some lipid molecules from the bilayer. This is qualitatively similar to the suggestion made in the literature for $\alpha$-helical peptides adsorbing on the lipid bilayer and the resulting membrane stress giving rise to the formation of pores. ${ }^{24}$ Only a small amount of lipid is removed consistent with the small amount of NP adsorption, and the lipid removal occurs leading to small pores or other forms of membrane defects that are not significant enough to perturb membrane stability.

\subsection{PMAA coated NPs adsorb more strongly to the bilayer}

QCM-D measurements of gold NP-bilayer interactions were conducted in the presence of polymethacrylic acid. One expects the spontaneous adsorption of PMAA over the gold NPs and therefore these experiments could reveal the interactions between the PMAA coated gold NPs and the lipid bilayer that had been exposed to PMAA. For $2 \mathrm{~nm}$ and $5 \mathrm{~nm}$ NPs, a small negative frequency change (mass increase) was observed, while the dissipation change was negligible (Fig.2 and Fig.3). For the $10 \mathrm{~nm}$ NPs, frequency and dissipation changes were both negligible, 
while for the $40 \mathrm{~nm}$ NPs there was a significant frequency increase (mass loss) accompanied by a small increase in dissipation. Since NP adsorption can perturb the lipids and cause some lipid removal, evidently the adsorption of NPs in the presence of PMAA must be larger to compensate for the mass loss. For the $10 \mathrm{~nm}$ and $40 \mathrm{~nm}$ NPs, other mechanisms should be operative (discussed in the next section) to cause the mass loss observed.

Studies on the interactions of PMAA with gel and liquid crystalline states of DMPC bilayer have been presented in the literature by Xie and Granick ${ }^{25}$. They found that a large amount of PMAA adsorbed even at the earliest measurement times, and for very low solution concentrations of PMAA $(0.1$ and $1 \mathrm{mg} / \mathrm{mL})$. This is similar to our QCM-D results where PMAA was found to adsorb on the PC bilayer from a $1 \mathrm{mg} / \mathrm{mL}$ solution of PMAA, giving rise to a frequency change of $24 \mathrm{~Hz}$ in about 2 minutes. Xie and Granick proposed that since the head groups of the DMPC were dipolar, being positive on the termini $\left(-\mathrm{N}\left(\mathrm{CH}_{3}\right)^{3+}\right)$ and negative a few angstroms underneath $\left(-\mathrm{PO}^{2-}\right)$, the ions of PMAA would electrostatically interact with the dipoles of the lipid head groups, with PMAA lying on the bilayer surface in the lipid head group region. They also concluded (using infrared measurements of $\mathrm{C}-\mathrm{H}$ vibration region) that the PMAA adsorption did not induce changes in the average area per phospholipid. The bilayer remained more or less unaffected. This is also consistent with the QCM-D observations where the observed dissipation changes remained very small, implying that the bilayer structure was not fundamentally affected. In the gel phase of the bilayer (not encountered in our QCM-D studies with PC) they found that defects exist (islands and patches of bilayer rather than homogeneous bilayer) and the PMAA adsorbs preferentially at the defect edges, thereby stabilizing the defects.

These observations suggest a simple interpretation for the small mass increase observed with the PMAA-coated 2 and 5-nm particles. The charge on the PMAA was small and the PMAA- 
coated nanoparticle had some hydrophobicity. As the PMAA adsorption on the bilayer created interactions between the small number of charges on the PMAA and multiple dipoles of the lipid head groups causing head group tilts and creating space for the surface adsorption of the PMAA coated nanoparticles. After the initial surface adsorption, the hydrophobic PMAA-coated nanoparticles could even penetrate into the bilayer as has been reported for small hydrophobic NPs through molecular dynamics simulations. This could also be represented as a larger partition coefficient $\mathrm{K}_{\text {lipw }}$ compared to the partition coefficient measured for the citric acid stabilized-gold NPs. The mass increase due to adsorption remained slightly larger than any mass loss due to removal of stressed lipids. Also any defects in the bilayer associated with the lipid removal can be filled by PMAA similar to the observation of Xie and Granick of PMAA adsorbing on defect edges.

The penetration of the pores by hydrophobic nanoparticles has been observed in molecular dynamics simulations where the particle is engulfed within the hydrophobic domain of the bilayer. For example, Li et al. used coarse-grained molecular dynamics simulations to show that the hydrophobicity of the nanoparticle controls the interaction with the membrane ${ }^{26}$. For NPs interacting with a dipalmitoylphosphatidylcholine (DPPC) membrane, a zwitterionic membrane that is similar to PC, Li et al. showed that hydrophobic NPs would become included in bilayer, while semi-hydropobic NPs adsorbed to the surface of the membrane ${ }^{26}$. When the simulation was allowed to progress for more time, deformations in the lipid bilayer were observed to be temporary, and rearrangement of lipid molecules could occur.

Experiments with lipid bilayers and vesicles have also shown relationships between NP physicochemical properties and interaction mechanisms with the lipid structure. For example, Bothun showed that hydrophobic $5.7 \mathrm{~nm} \mathrm{Ag}$ nanoparticles, functionalized with decanethiol, 
became internalized in the hydrophobic lipid interior of a DPPC liposome ${ }^{27}$. The NP diameter was near or exceeding the bilayer thickness, but due to the hydrophobic properties of the golddecanethiol NPs, the particles could insert into the hydrophobic region of the bilayer. Other examples of gold and silver NPs treated with hydrocarbons to impart hydrophobicity have

demonstrated NP insertion into vesicles and/or lipid bilayers ${ }^{28-30}$. In order to embed a nanoparticle into a hydrophobic membrane, the NP must be small and hydrophobic, with sizes estimated to be $<4-8 \mathrm{~nm}^{27,31}$.

\subsection{Size-dependent NP engulfment by bilayer causes lipid removal}

For $40 \mathrm{~nm}$ NPs coated with PMAA, large mass losses were observed. We have attributed the mass increase observed for the 2 and $5 \mathrm{~nm}$ NPs to the stronger adhesion between the PMAA coated gold NPs and the bilayer. The question we need to answer is why such strong adhesion lead to mass loss in the case of the $40 \mathrm{~nm}$ NPs, but not for the smaller NPs. In addition, adhesion plays a different role in the behavior of the $40 \mathrm{~nm}$ NPs in water compared to with PMAA. We propose that the large PMAA-coated NP was engulfed by a fragment of the bilayer (Fig.6) causing a significant amount of lipid to be removed from the bilayer (hence, the observed mass loss), and that this engulfment does not occur for the smaller PMAA-coated NPs and for the citric acid-stabilized $40 \mathrm{~nm}$ NPs in water.

To determine the likelihood that the NP could be engulfed by the lipid bilayer, one has to consider the free energy change between the nanoparticle-engulfed state and the initial state of the planar bilayer coexisting with the spherical particle. This free energy change should be negative for this engulfment process to occur spontaneously. Taking a phenomenological view, three principal free energy contributions are to be considered. The first is the interaction of the NP with the bilayer in contact with it. Second, the lipid that was present in the planar bilayer is 
now present in a spherical bilayer. Finally, the removal of the lipid from the planar bilayer leaves behind a lipid interface in contact with the aqueous domain. We represent these three contributions through the simple expression

$$
\Delta g=-4 \pi R_{P}^{2} \epsilon_{a d h}+8 \pi \kappa+4 \pi R_{P} \delta \epsilon_{i n t}
$$

where $\Delta g$ is the free energy change expressed per nanoparticle, $R_{P}$ is the radius of the nanoparticle, $-\varepsilon_{a d h}$ is the attractive adhesive interaction energy per unit area between the nanoparticle and the bilayer engulfing it, $\kappa$ is the bending modulus of the bilayer, $\delta$ is the thickness of the bilayer and $\varepsilon_{i n t}$ is the positive interfacial energy per unit area. The first term represents the adhesion energy between the fully engulfed PMAA coated NP and the bilayer. We could instead have considered only partial engulfment, but the qualitative discussions and conclusions below are not affected by this choice. The second term represents the bending energy associated with the planar bilayer becoming a spherical bilayer. The third term represents the free energy of formation of the interface, or the edge energy. The interfacial area is taken as equal to $4 \pi R_{P} \delta$, corresponding to a pore of radius $2 R_{P}$ (based on the consideration that the pore area is equal to the surface area of a particle since the lipid from this pore is covering the entire surface of the particle). The condition $\Delta g<0$ for the engulfment of the nanoparticle by the bilayer is satisfied if the NP size exceeds a critical particle radius $R_{P C}$ obtained by equating $\Delta g$ to zero.

$$
2 R_{P C}=\delta \frac{\varepsilon_{\text {int }}}{\varepsilon_{\text {adh }}}+\left[\left(\delta \frac{\varepsilon_{\text {int }}}{\varepsilon_{\text {adh }}}\right)^{2}+\frac{8 \kappa}{\varepsilon_{\text {adh }}}\right]^{1 / 2}
$$

The calculated critical particle diameter $\left(2 R_{P C}\right)$ is plotted in Fig.7 for various values of the adhesion energy and interfacial energy. In all calculations, the bending modulus of the bilayer is taken equal to $20 \mathrm{kT}$ as has been reported in the literature. ${ }^{32}$ Obviously, the engulfment is more 
probable for larger NPs than smaller NPs, since the energy penalty associated with the bilayer bending decreases with increasing particle size. Further, if the adhesive energy $\varepsilon_{a d h}$ is large or if the interface energy $\varepsilon_{i n t}$ is small, then the critical particle radius decreases, implying that smaller NPs could be engulfed by the bilayer. Comparing the PMAA coated NPs of the smaller size against the $40 \mathrm{~nm} \mathrm{NPs}$, all of which have the same adhesion energy and interfacial energy, this model clearly suggests why the larger size particle allowed for engulfment and resulted in mass removal from the bilayer because of the bending energy contribution. Comparing the $40 \mathrm{~nm}$ citric acid-stabilized particle against the PMAA-coated NP, this model clearly suggests how the stronger adhesion in the latter case permits engulfment by the bilayer with resultant mass removal.

\subsection{Delayed PMAA adsorption on bilayer defects}

For the $40 \mathrm{~nm}$ PMAA coated particles, we observed a mass loss during the beginning of stage (e) followed by a mass increase during the beginning of stage (f). Since PMAA can adsorb on the defect sites, it is possible to interpret our results in terms of PMAA adsorption on the defect sites on the bilayer where the lipid had been removed. However, the adsorption of PMAA did not occur immediately following lipid removal early on in stage (e) even though the PMAA in the PMAA + NP + water solvent was available for adsorption. Instead, the PMAA adsorption process became possible only after the end of PMAA $+\mathrm{NP}+$ water solvent flow (stage e) and when the flow of PMAA + water commenced (beginning of stage f). We speculate that this could be due to the diffusional barrier provided by the presence of NPs near the bilayer interface preventing the PMAA from reaching and adsorbing onto the bilayer and bilayer defects. Some support for such a speculation comes from a recent study that investigated the role of gravity force on spherical gold NPs, surface modified with 3-mercaptopropionic acid, interacting with 
lipid bilayer. ${ }^{33}$ Zhu et al used the QCM-D with a closed flow chamber, positioning the supported lipid bilayer at the bottom and top of the liquid medium by adopting an upright and inverted configuration, respectively. On the upright crystal, the NP solution was above the bilayer while in the inverted crystal, the solution was below the bilayer. They found larger nanoparticle adsorption on the upright bilayer compared to the inverted bilayer. This could not be attributed to the gravity force since it is much smaller in magnitude compared to van der Waals forces and electrostatic forces governing adsorption. They argued that the gravity force causes a gradient in the NP distribution in the liquid in contact with the bilayer. For the upright bilayer, such gravity induced sedimentation causes a high local concentration of the NP near the bilayer and that is responsible for the increased adsorption. For the inverted crystal, the opposite situation prevails.

Based on this study, we speculate that a higher local concentration of the $40 \mathrm{~nm}$ gold NPs exists close to the bilayer surface preventing the PMAA from diffusing to and adsorbing on the bilayer during stage (e). Once the NP flow ends, this barrier is removed and even at the very beginning of stage (f) PMAA could immediately adsorb on the defect sites of the bilayer adding to the mass. Additional measurements and other experimental methods would be needed to confirm this speculative explanation.

\section{CONCLUSIONS}

Nanomaterials exhibit cytotoxicity through various mechanisms. All of the mechanisms except cell membrane disruption can result also from the molecular, atomic, or ionic species constituting the NP, without requiring the nanoparticle nature of the material. Supported lipid bilayer membranes represent an attractive model membrane platform to study membrane disruption, since there are no interfering or complicating factors such as would be the case if a biological cell is used. In this work, QCM-D was employed to study the interactions of gold NPs 
with a zwitterionic egg PC bilayer both in the absence of and in the presence of polymethacrylic acid acting as a NOM simulant. The results show that $2,5,10$, and $40 \mathrm{~nm}$ diameter citric acidstabilized gold NPs caused a small lipid loss from the bilayer. The dissipation changes were small enough to suggest that no significant perturbation of the membrane structure occurred. Since the lipid loss was quite small, it is possible that pores of a size sufficient to permeabilize the membrane were not generated, implying that the NPs were not cytotoxic. NP adsorption induces stress on the lipids, causing some lipid removal as the stress relief or free energy lowering mechanism. When the NPs were in the presence of PMAA, the smaller NPs caused a mass increase on the bilayer. The increased adhesion between the NPs and the bilayer due to PMAA was responsible for increased NP adsorption overcompensating for the loss of some lipid molecules. The most remarkable change observed in this study was the significant mass loss in the case of the $40 \mathrm{~nm} \mathrm{NP}$, in the presence of PMAA. We attributed this to the lipid bilayer engulfing the NP and being removed from the crystal surface. To rationalize the size dependent behavior in the presence of PMAA and also the difference in behavior between the presence and absence of PMAA, we proposed a simple model for NP-polymer-bilayer interactions accounting for the competition between the particle-bilayer adhesion energy, the bilayer bending energy and the interfacial energy at bilayer defect edges. The model clearly shows that for all particle sizes, if the particle adhesion is not adequate, then lipid engulfment of NP does not occur. In contrast, if the adhesion is stronger, then particles of larger size allow for lipid engulfment but not particles of smaller sizes. The model thus rationalizes the observed behavior of $40 \mathrm{~nm}$ NP in the presence of PMAA wherein the more adhesive NPs are engulfed by the bilayer and leave the crystal surface causing significant mass loss. This large mass change can be associated with membrane disruption and cytotoxicity. This suggests that even if gold NPs are intrinsically not 
cytotoxic, they can become cytotoxic in the presence of organic additives representing NOM because of the changes in the adhesive interactions promoted by the adsorbing polymer. This result would suggest that nanotoxicology studies should focus not only on the nanoparticle and its surface chemistries but also on the organic species present in the environment in order to determine the risk of nanoparticle cytotoxicity. 


\section{Corresponding Author}

*Terri A. Camesano, Department of Chemical Engineering, Worcester Polytechnic Institute, 100 Institute Rd. Worcester, MA 01609; Phone: 508-831-5380; Fax: 508-831-5853; Email: terric@,wpi.edu

*Ramanathan Nagarajan, Molecular Sciences and Engineering Team, Natick Soldier Research, Development \& Engineering Center, 15 Kansas Street, Natick MA 01760; Phone: 508-233-6445; Fax: 508-233-4469; E-mail: Ramanathan.Nagarajan.Civ@mail.mil

\section{Author Contributions}

The manuscript was written through contributions of all authors. All authors have given approval to the final version of the manuscript.

\section{Funding Sources}

National Science Foundation (CBET 0966496)

\section{ACKNOWLEDGMENT}

This work was supported in part by the National Science Foundation (CBET 0966496). The authors thank Geoffrey Bothun at the University of Rhode Island for helpful discussions. In addition, Joseph Duffy, Christopher Lambert, Reeta Prusty Rao, and Hong Susan Zhou at Worcester Polytechnic Institute assisted with some use of lab equipment. Several WPI students assisted with preliminary experiments in this area: Andrew Carey, Houssam Lazkhani, Thomas Finelli, Yan Yan, and Sophia D’Angelo. Support from Natick Soldier Research, Development and Engineering Center to one of the authors $(\mathrm{RN})$ is acknowledged. 


\section{ABBREVIATIONS}

NOM, natural organic matter; NP, nanoparticle; PC, L- $\alpha$-phosphatidylcholine (egg, chicken);

PMAA, polymethacrylic acid; QCM-D, quartz crystal microbalance with dissipation; ROS, reactive oxygen species; SLB, supported lipid bilayer; 


\section{REFERENCES}

[1] C. R. Thomas, S. George, A. M. Horst, Z. Ji, R. J. Miller, J. R. Peralta-Videa, T. Xia, S. Pokhrel, L. Mädler, J. L. Gardea-Torresdey, P. A. Holden, A. A. Keller, H. S. Lenihan, A. E. Nel, J. I. Zink, Nanomaterials in the environment: From materials to high-throughput screening to organisms, ACS Nano 5 (2011) 13-20.

[2] N. Lewinski, V. Colvin, R. Drezek, Cytotoxicity of nanoparticles, Small 4 (2008) 26-29.

[3] A. Nel, T. Xia, L. Mädler, N. Li, Toxic potential of materials at the nanolevel, Science 311 (2006) 622-627.

[4] L. K. Limbach, P. Wick, R. Manser, R. N. Grass, A. Bruinink, W. J. Stark, Exposure of engineered nanoparticles to human lung epithelial cells: influence of chemical composition and catalytic activity on oxidative stress, Environ. Sci. Technol. 41 (2007) 4158-4163.

[5] L. Braydich-Stolle, S. Hussain, J. J. Schlager, M. C. Hoffman, In vitro cytotoxicity of nanoparticles in mammalian germline stem cells, Toxicol. Sci. 88 (2005) 412-419.

[6] A. Nebbioso, A. Piccolo, Basis of a humeomics science: Chemical fractionation and molecular characterization of humic biosuprastructures, Biomacromolecules 12 (2011) $1187-1199$.

[7] J. Gao, K. Powers, Y. Wang, H. Zhou, S. M. Roberts, B. M. Moudgil, B. Koopman, D. S. Barber, Influence of Suwannee River humic acid on particle properties and toxicity of silver nanoparticles, Chemosphere 89 (2012) 96-101.

[8] R. F. Domingos, N. Tufenkji, K. I. Wilkinson, Aggregation of titanium dioxide nanoparticles: role of a fulvic acid, Environ. Sci. Technol. 43 (2009) 1282-1286. 
[9] A. Czogalla, M. Grzybek, W. Jones, Ü. Coskun, Validity and applicability of membrane model systems for studying interactions of peripheral membrane proteins with lipids, Biochim. Biophys. Acta 1841 (2014) 1049-1059.

[10] H. Liu, D. Chen, L. Li, T. Liu, L. Tan, X. Wu, F. Tang, Multifunctional gold nanoshells on silica nanorattles: A platform for the combination of photothermal therapy and chemotherapy with low systemic toxicity, Angew. Chem. Int. Ed. Engl. 50 (2011) 891-895.

[11] A. Sharma, A. Tandon, J. C. Tovey, R. Gupta, J. D. Robertson, J. A. Fortune, A. M. Klibanov, J. W. Cowden, F. G. Rieger, R. R. Mohan, Polyethylenimine-conjugated gold nanoparticles: Gene transfer potential and low toxicity in the cornea, Nanomedicine 7 (2011) 505-513.

[12] M. Auffan, J. Rose, J. Y. Bottero, G. V. Lowry, J. P. Jolivet, M. R. Wiesner, Towards a definition of inorganic nanoparticles from an environmental, health and safety perspective, Nat. Nano 4 (2009) 634-641.

[13] Y. Barenholz, D. Gibbes, B. J. Litman, J. Goll, T. E. Thompson, R. D. Carlson, A simple method for the preparation of homogeneous phospholipid vesicles, Biochemistry 16 (1977) 2806-2810.

[14] C. A. Keller, K. Glasmästar, V. P. Zhdanov, B. Kasemo, Formation of supported membranes from vesicles, Phys. Rev. Lett. 84 (2000) 5443-5446.

[15] B. Y. Moghadam, W. C. Hou, C. Corredor, P. Westerhoff, J. D. Posner, Role of nanoparticle surface functionality in the disruption of model cell membranes, Langmuir 28 (2012) 16318-16326. 
[16] A. Mechler, S. Praporski, K. Atmuri, M. Boland, F. Separovic, L. L. Martin, Specific and selective peptide-membrane interactions revealed using quartz crystal microbalance, Biophys. J. 93 (2007) 3907-3916.

[17] M. Rodahl, F. Höök, C. Fredriksson, C. A. Keller, A. Krozer, P. Brzezinski, M. Voinova, B. Kasemo, Simultaneous frequency and dissipation factor QCM measurements of biomolecular adsorption and cell adhesion, Faraday Discuss. 107 (1997) 229-246.

[18] M. V. Voinova, M. Jonson, B. Kasemo, 'Missing mass' effect in biosensor's QCM applications, Biosens. Bioelectron. 17 (2002) 835-841.

[19] K. F. Wang, R. Nagarajan, T. A. Camesano, Antimicrobial peptide alamethicin insertion into lipid bilayer: A QCM-D exploration, Colloids Surf. B: Biointerfaces 116 (2014) 472481.

[20] A. Einstein, Eine neue bestimmung der moleküldimensionen, Ann. Phys. 324 (1906) 289306.

[21] J. A. Gallego-Urrea, J. Tuoriniemi, M. Hassellöv, Applications of particle-tracking analysis to the determination of size distributions and concentrations of nanoparticles in environmental, biological and food samples, Trends Anal. Chem. 30 (2011) 473-483.

[22] W. C. Hou, B. Y. Moghadam, C. Corredor, P. Westerhoff, J. D. Posner, Distribution of functionalized gold nanoparticles between water and lipid bilayers as model cell membranes, Environ. Sci. Technol. 46 (2012) 1869-1876.

[23] T. J. Zwang, W. R. Fletcher, T. J. Lane, M. S. Johal, Quantification of the layer of hydration of a supported lipid bilayer, Langmuir 26 (2010) 4598-4601.

[24] H. W. Huang, F. Y. Chen, M. T. Lee, Molecular mechanism of peptide-induced pores in membranes, Phys. Rev. Lett. 92 (2004) 198304. 
[25] A. F. Xie, S. Granick, Phospholipid membranes as substrates for polymer adsorption, Nat Mater. 1 (2002) 129-133.

[26] Y. Li, X. Chen, N. Gu, Computational investigation of interaction between nanoparticles and membranes: hydrophobic/hydrophilic effect, J. Phys. Chem. B 112 (2008) 1664716653.

[27] G. D. Bothun, Hydrophobic silver nanoparticles trapped in lipid bilayers: Size distribution, bilayer phase behavior, and optical properties, J. Nanobiotechnol. 6 (2008) 13.

[28] S. H. Park, S. G. Oh, J. Y. Mun, S. S. Han, Effects of silver nanoparticles on the fluidity of bilayer in phospholipid liposome, Colloids Surf. B: Biointerfaces 44 (2005) 117-122.

[29] S. H. Park, S. G. Oh, J. Y. Mun, S. S. Han, Loading of gold nanoparticles inside the DPPC bilayers of liposome and their effects on membrane fluidities, Colloids Surf. B: Biointerfaces 48 (2006) 112-118.

[30] H. Jang, L. E. Pell, B. A. Korgel, D. S. English, Photoluminescence quenching of silicon nanoparticles in phospholipid vesicle bilayers, J. Photochem. Photobiol. A: Chem. 158 (2003) 111-117.

[31] M. Schulz, A. Olubummo, W. H. Binder, Beyond the lipid-bilayer: Interaction of polymers and nanoparticles with membranes, Soft Matter 8 (2012) 4849-4864.

[32] E. Evans, W. Rawicz, Entropy-driven tension and bending elasticity in condensed-fluid membranes, Phys. Rev. Lett. 64 (1990) 2094-2097.

[33] T. Zhu, Z. Jiang, Y. Ma, Adsorption of nanoparticles and nanoparticle aggregates on membrane under gravity, Appl. Phys. Lett. 102 (2013) 153109. 


\section{Figure Captions}

Figure 1. Representative plots showing QCM-D frequency and dissipation changes as a function of time. The blue lines represent frequency and the red lines represent dissipation. Overtones 3 , $5,7,9$, and 11 are shown. Common to all plots is the formation of the PC bilayer on the $\mathrm{SiO} 2$ crystal, depicted by the large frequency decrease as vesicles adsorbed, followed by a rapid frequency increase as the vesicles ruptured. Buffer was then flowed through the system to stabilize the bilayer by removing un-ruptured or excess vesicles. (A) $10 \mathrm{~nm}$ gold NPs in water. (B) $40 \mathrm{~nm}$ gold NPs in water. For A and B, following the bilayer formation, the time (in minutes) for each step was: buffer rinse 7:20; water rinse 15:45; gold NPs 24:17; water rinse 34:37; buffer rinse 42:53. (C) $10 \mathrm{~nm}$ gold NPs in PMAA solution. (D) $40 \mathrm{~nm}$ gold NPs in PMAA solution. For $\mathrm{C}$ and $\mathrm{D}$, the time for each step was: buffer rinse 9:25; water rinse 15:52; PMAA solution 23:35; gold NPs in PMAA 32:02; PMAA solution 42:27; water rinse 50:50; buffer rinse 59:04.

Figure 2. Dissipation changes associated with the nanoparticle-bilayer interactions for gold NPs of size (A) $2 \mathrm{~nm}$; (B) $5 \mathrm{~nm}$; (C) $10 \mathrm{~nm}$; and (D) $40 \mathrm{~nm}$. Blue bars are for NPs in water, and pink are for NPs in PMAA solution. Error bars show the standard error values. Each data point is based on at least 5 experimental samples at a constant concentration of $7.14 \times 10^{10}$ particles/mL. Statistical analysis was performed with SigmaPlot 12.5 software at a $95 \%$ confidence interval $(\alpha=0.05)$. The results of one-way analysis of variance (ANOVA) test showed that the results of different sizes have statistically significant difference $(\mathrm{P}=<0.001)$.

Figure 3. Mass changes estimated from Sauerbrey relationship for interactions of gold NPs with PC bilayer, for NPs of size (A) $2 \mathrm{~nm}$; (B) $5 \mathrm{~nm}$; (C) $10 \mathrm{~nm}$; and (D) $40 \mathrm{~nm}$. Blue bars are for NPs in water, and pink are for NPs in PMAA solution. Error bars show the standard error values. 
Each data point reported is based on at least 5 experimental samples at a constant concentration of $7.14 \times 10^{10}$ particles $/ \mathrm{mL}$. Statistical analysis was performed with SigmaPlot 12.5 software at a $95 \%$ confidence interval $(\alpha=0.05)$. The results of one-way analysis of variance (ANOVA) test showed that the results of different sizes have statistically significant difference $(\mathrm{P}=<0.001)$.

Figure 4. Frequency and dissipation changes associated with the PMAA-bilayer interactions both before ( $\mathrm{A}$ and $\mathrm{B}$ ) and after $(\mathrm{C}$ and $\mathrm{D})$ the exposure of the bilayer to the nanoparticles. Patterned bars are for $10 \mathrm{~nm}$ particles and the filled bars are for the $40 \mathrm{~nm}$ particles. Note that (A) and (B) represent data before nanoparticle exposure occurred in QCM-D runs done with the specified nanoparticle sizes. Error bars show the standard error values. Each data point is based on at least 5 experimental samples at a constant concentration of $7.14 \times 10^{10}$ particles $/ \mathrm{mL}$. Statistical analysis was performed with SigmaPlot 12.5 software at a $95 \%$ confidence interval $(\alpha=0.05)$. The results of one-way analysis of variance (ANOVA) test showed that the results of different sizes have statistically significant difference $(\mathrm{P}=<0.001)$.

Figure 5. Schematic view of citric acid-stabilized gold nanoparticles interacting with the supported lipid bilayer. The nanoparticles adsorb on the surface displacing lipid head groups. This causes the lipids to change their conformation, making their energy states higher than that of the average lipid on the bilayer. The resulting stress on the bilayer is relieved by the removal of some lipid molecules from the bilayer. The lipid loss may create small pores in the bilayer. The mass loss from lipid removal outweighs the mass addition from the adsorbed nanoparticles and net mass loss is recorded by the QCM-D.

Figure 6. Schematic view of PMAA coated gold nanoparticles interacting with the supported lipid bilayer. For the $40 \mathrm{~nm}$ nanoparticle, the adhesive interaction of the nanoparticle with the 
bilayer allows a fragment of the bilayer to partially or completely engulf the nanoparticle and leave the supported bilayer. This appears as a significant mass loss recorded by the bilayer. For the 2, 5 and $10 \mathrm{~nm}$ PMAA-coated nanoparticles, such engulfment is not favored as discussed in detail in the text.

Figure 7. Calculated critical nanoparticle diameter for engulfment by the lipid bilayer as a function of the adhesive interactions between the nanoparticle and the bilayer and the interfacial energy of the bilayer at the defect edge. The critical diameter of the nanoparticle at which engulfment can occur becomes smaller with increasing adhesive energy and decreasing interfacial energy. The calculations are based on a phenomenological free energy model discussed in the text. 


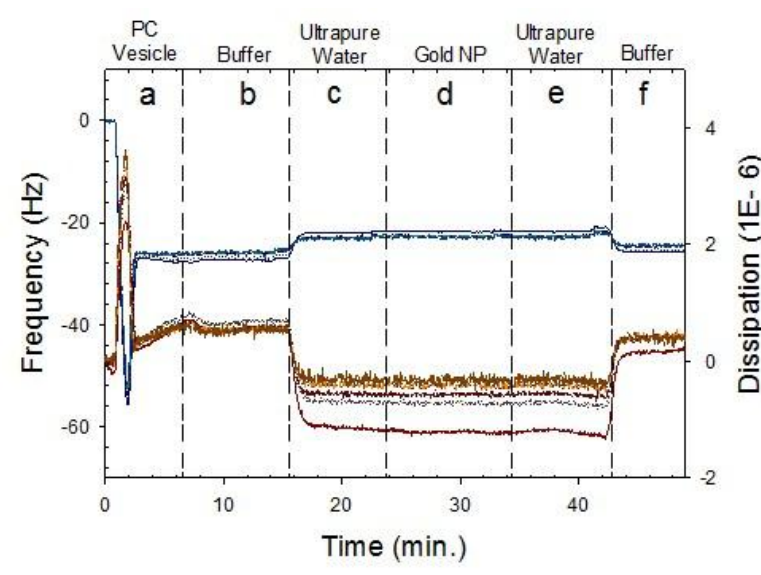

A. $10 \mathrm{~nm}$ NP- Water

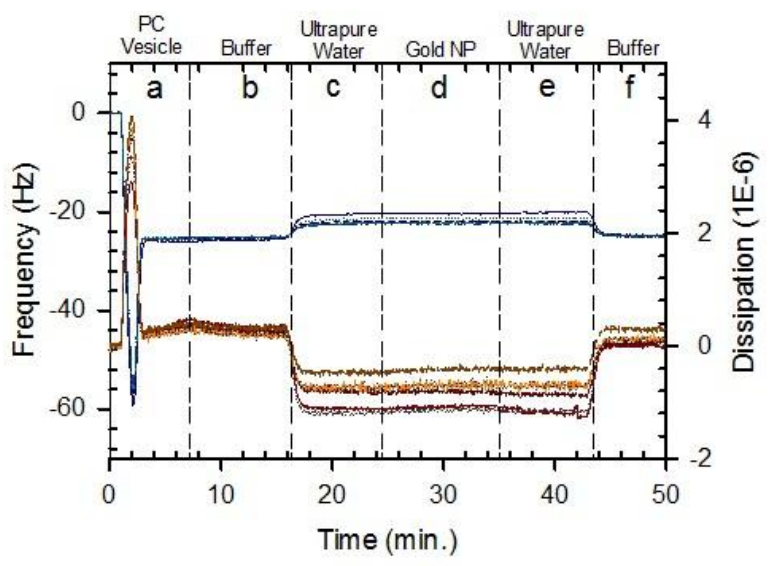

B. $40 \mathrm{~nm}$ NP- Water

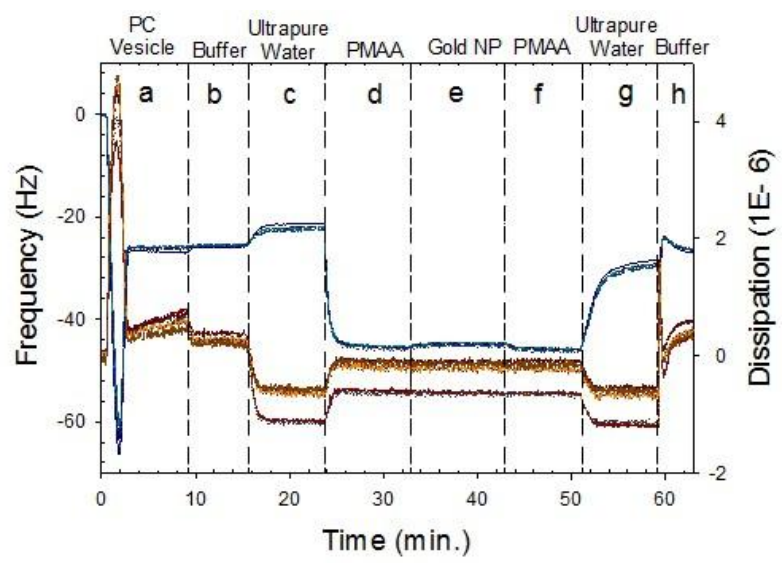

C. $10 \mathrm{~nm}$ NP - PMAA

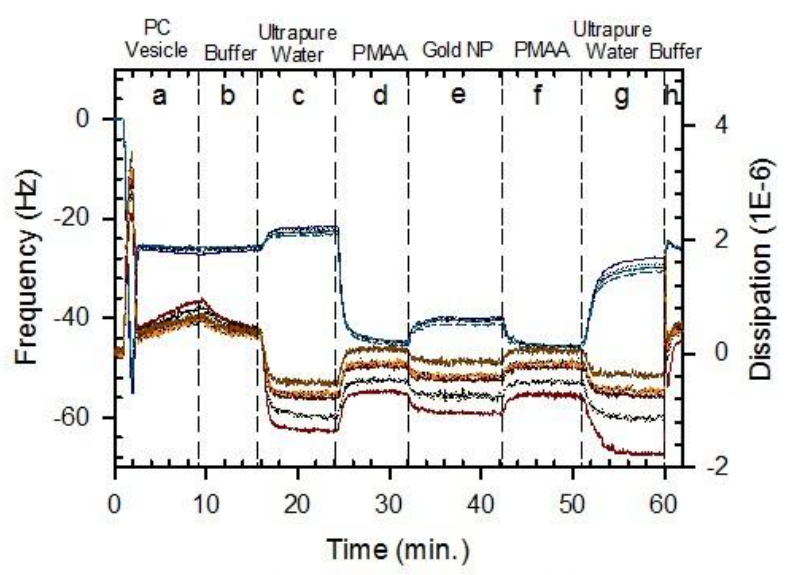

D. $40 \mathrm{~nm}$ NP - PMAA

Figure 1 


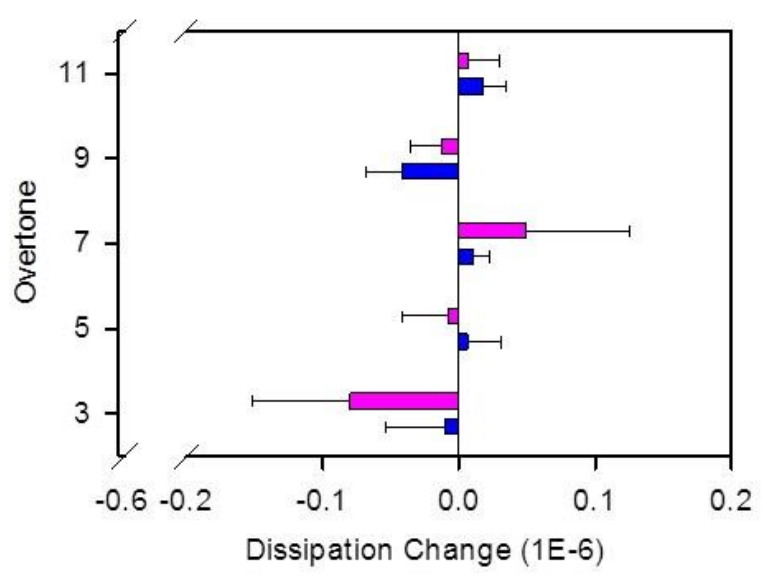

A. $2 \mathrm{~nm} \mathrm{NP}$

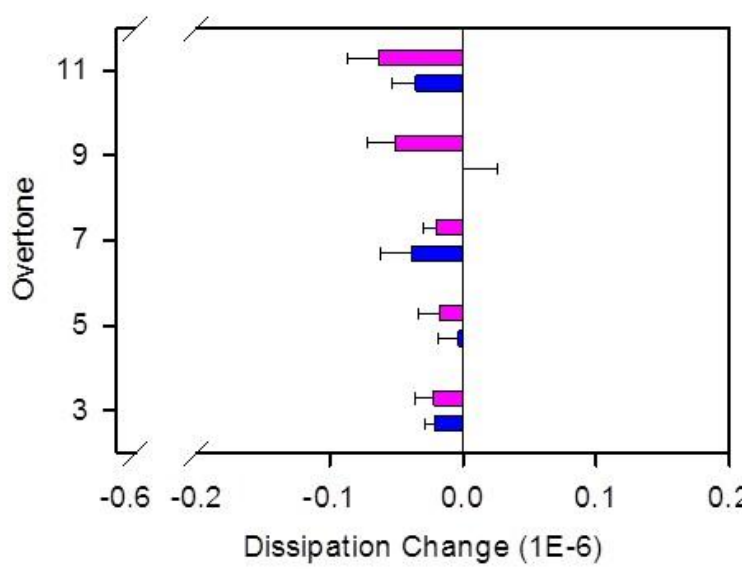

C. $10 \mathrm{~nm}$ NP

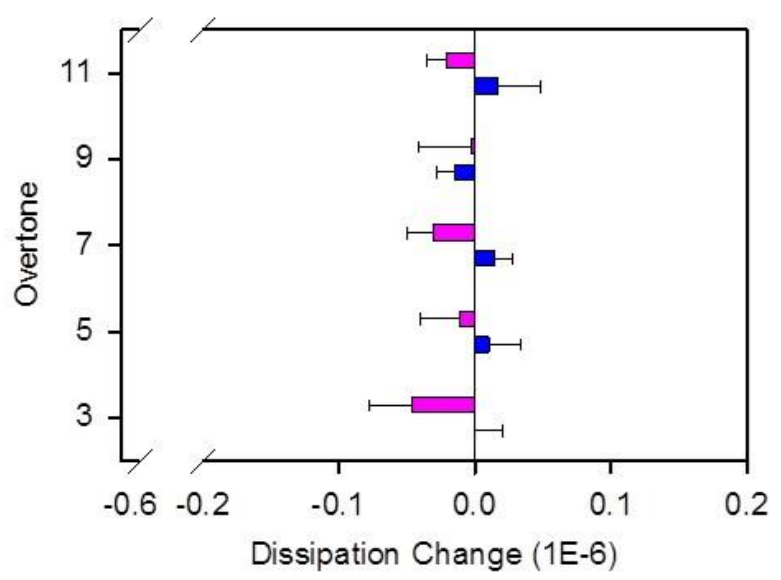

B. $5 \mathrm{~nm}$ NP

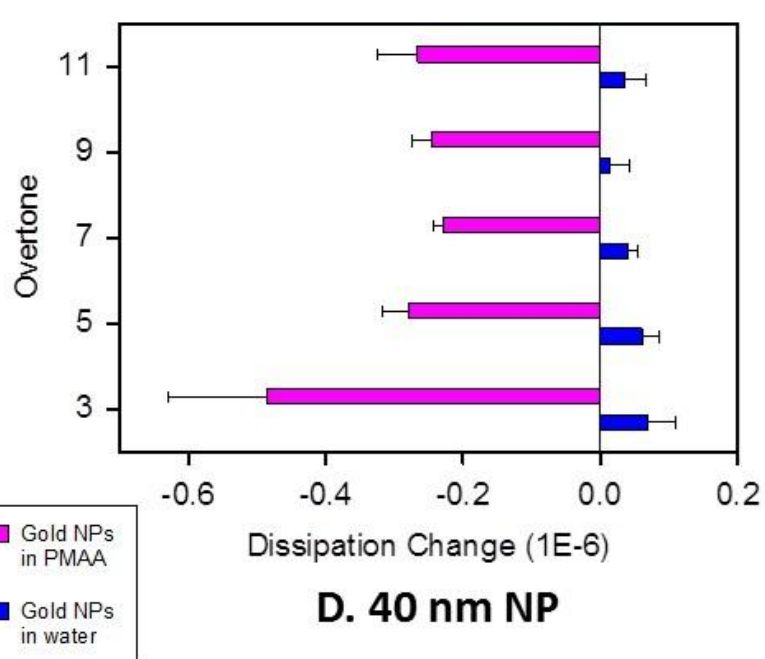

Figure 2 


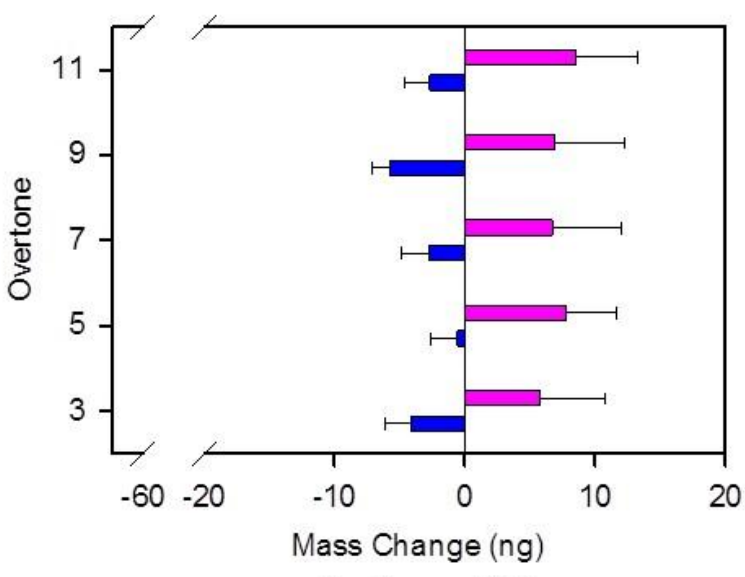

A. $2 \mathrm{~nm} \mathrm{NP}$
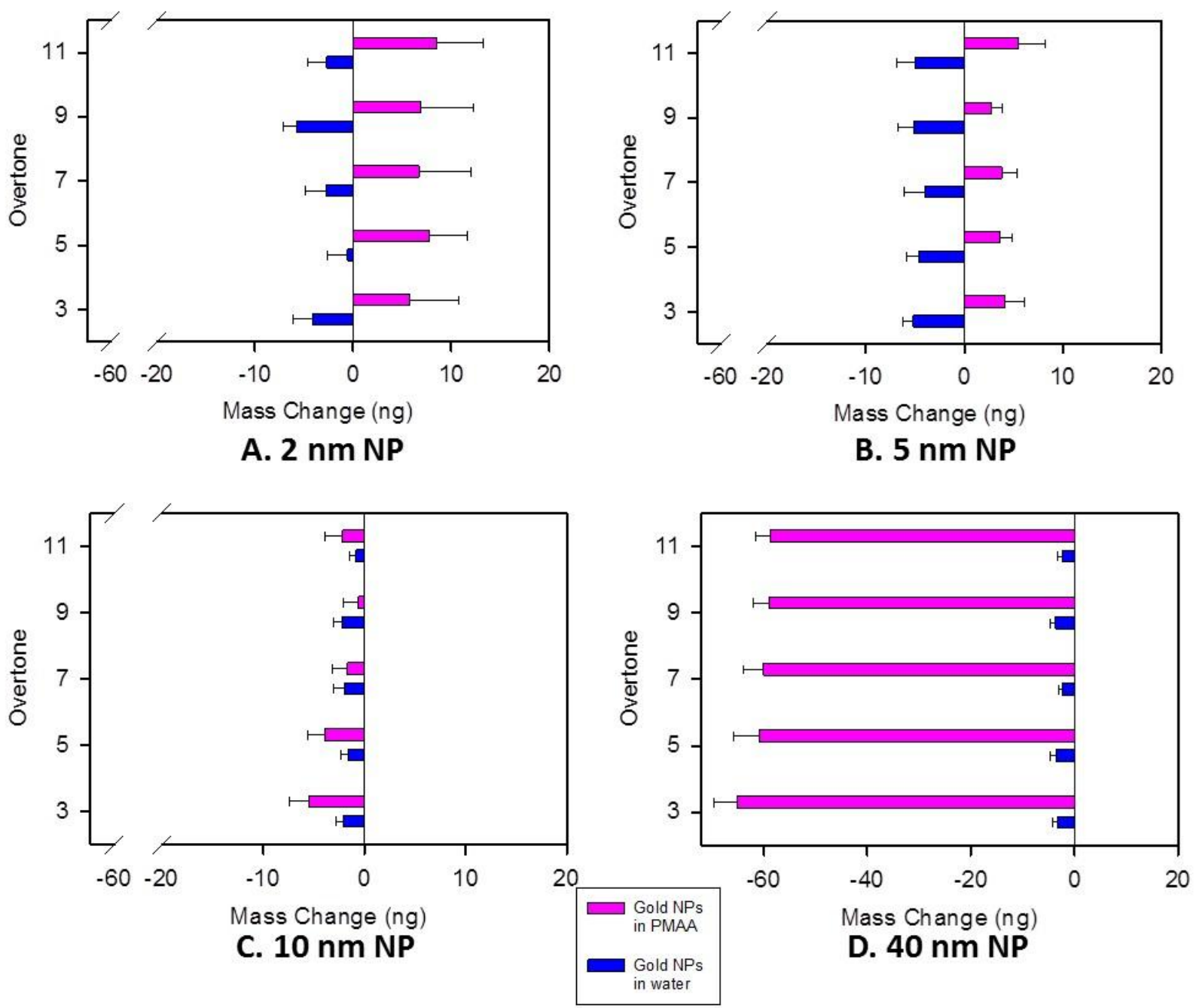

B. $5 \mathrm{~nm}$ NP

Figure 3 


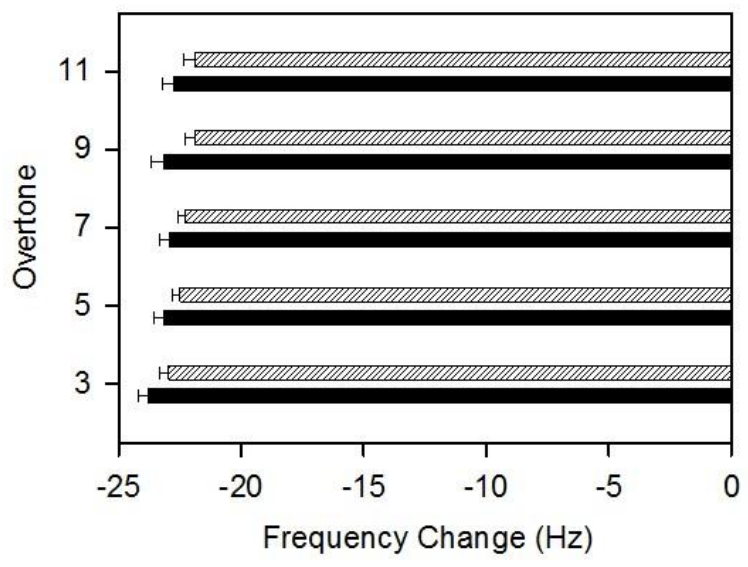

A. Process (c) to (d)

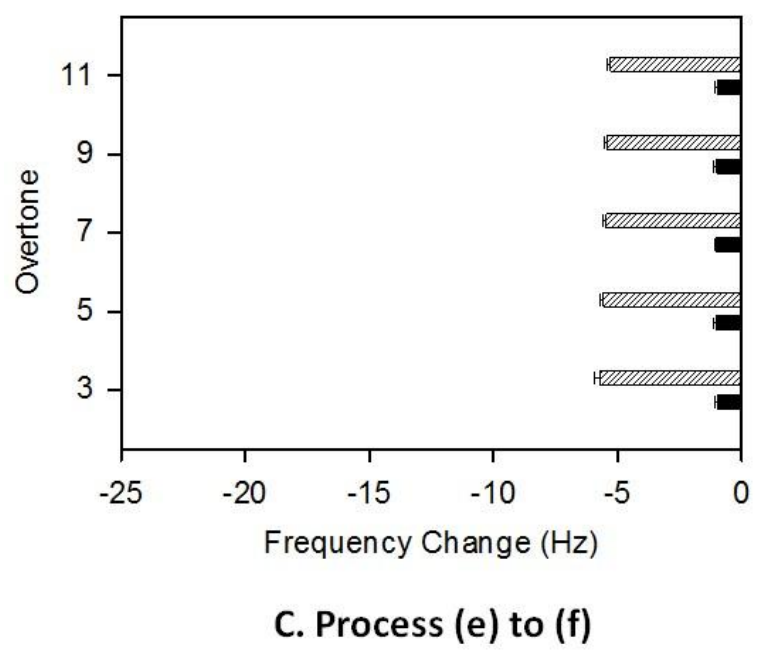

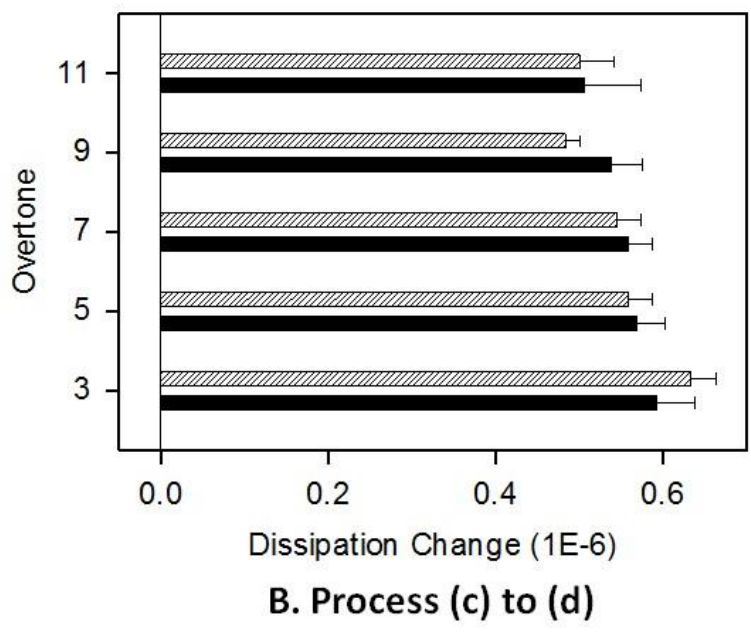

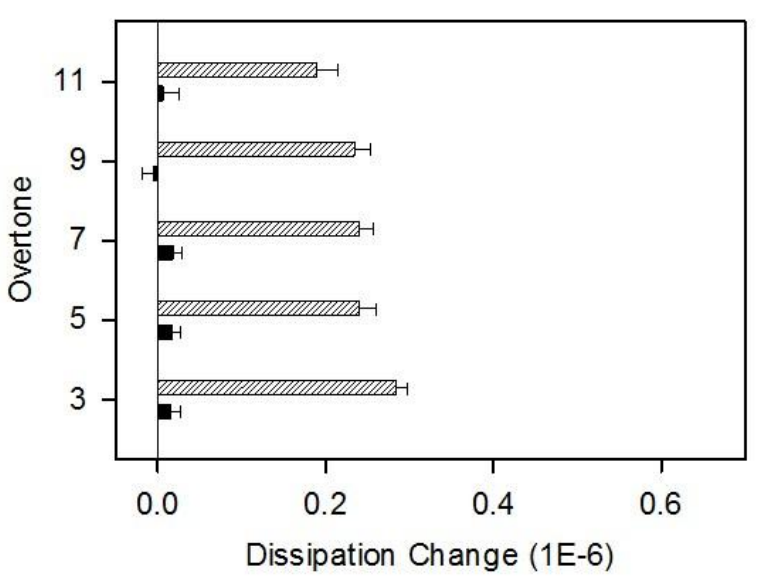

D. Process (e) to (f)

Figure 4 


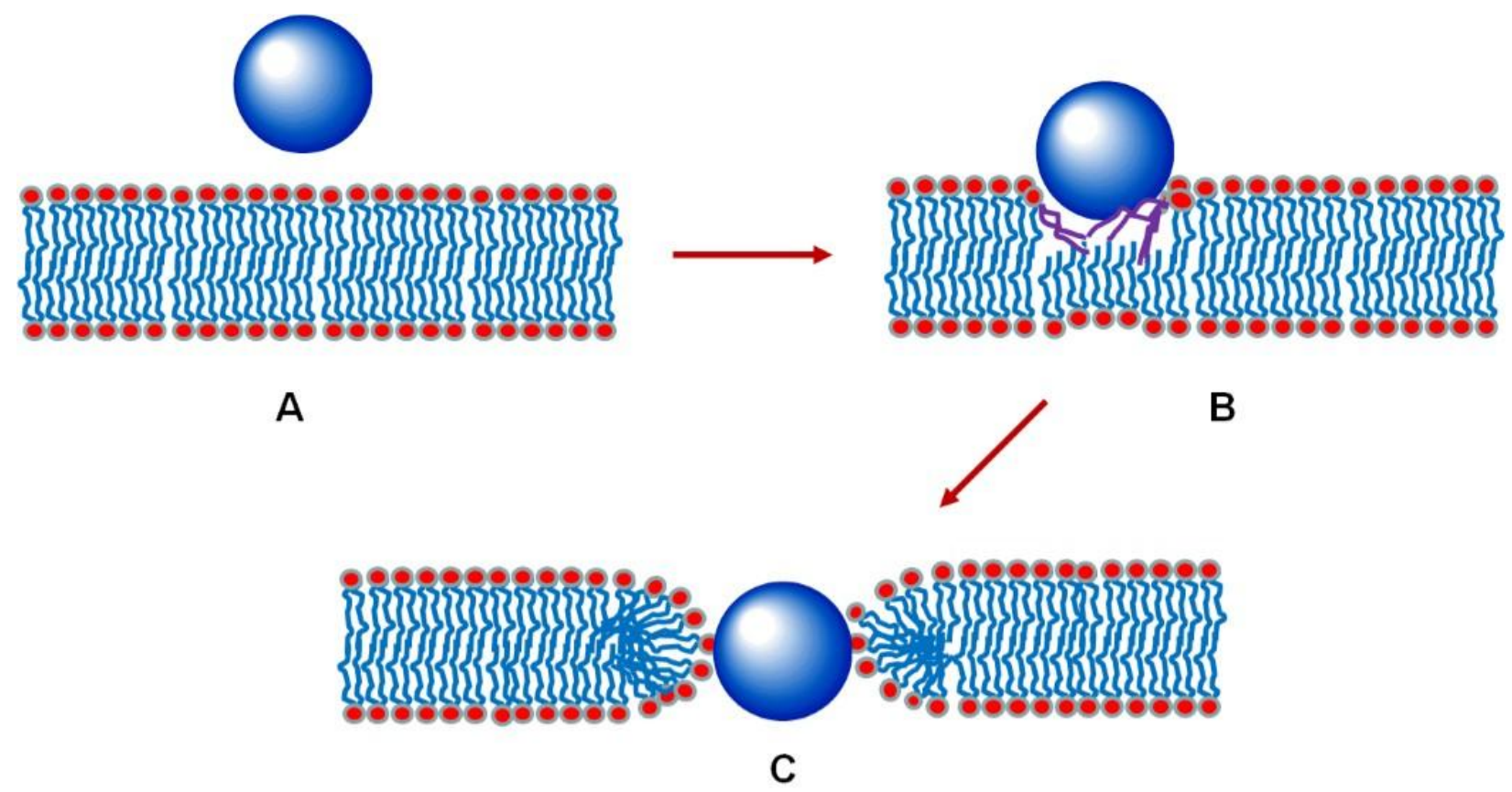

Figure 5 

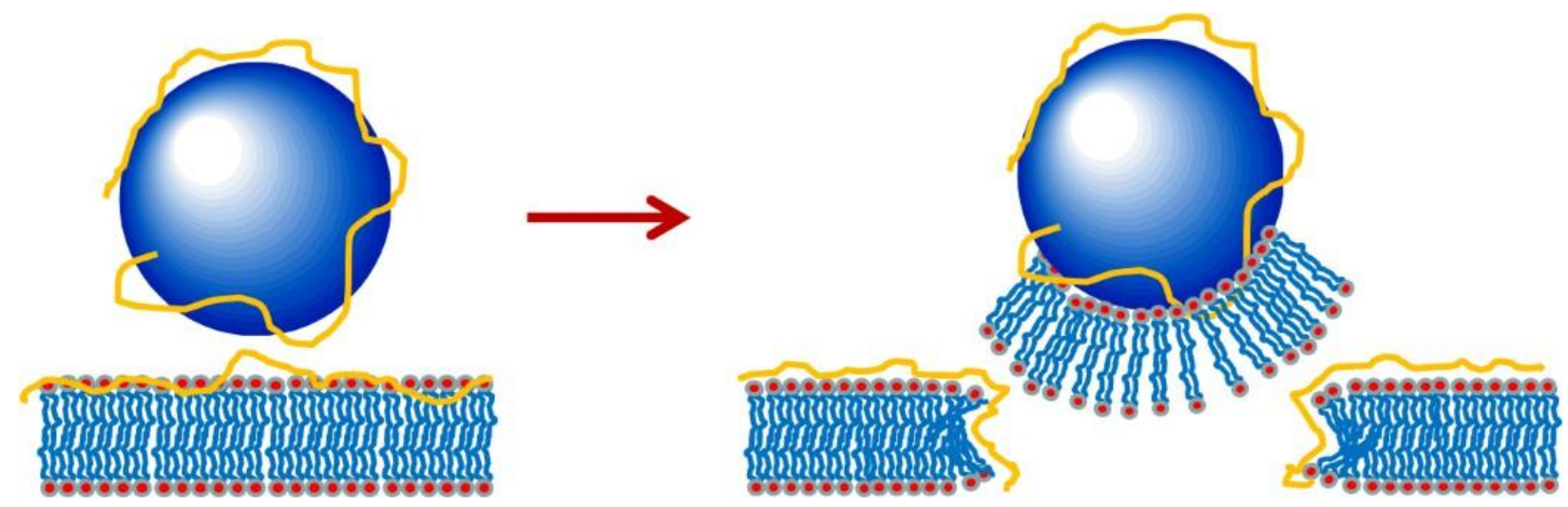

Figure 6 


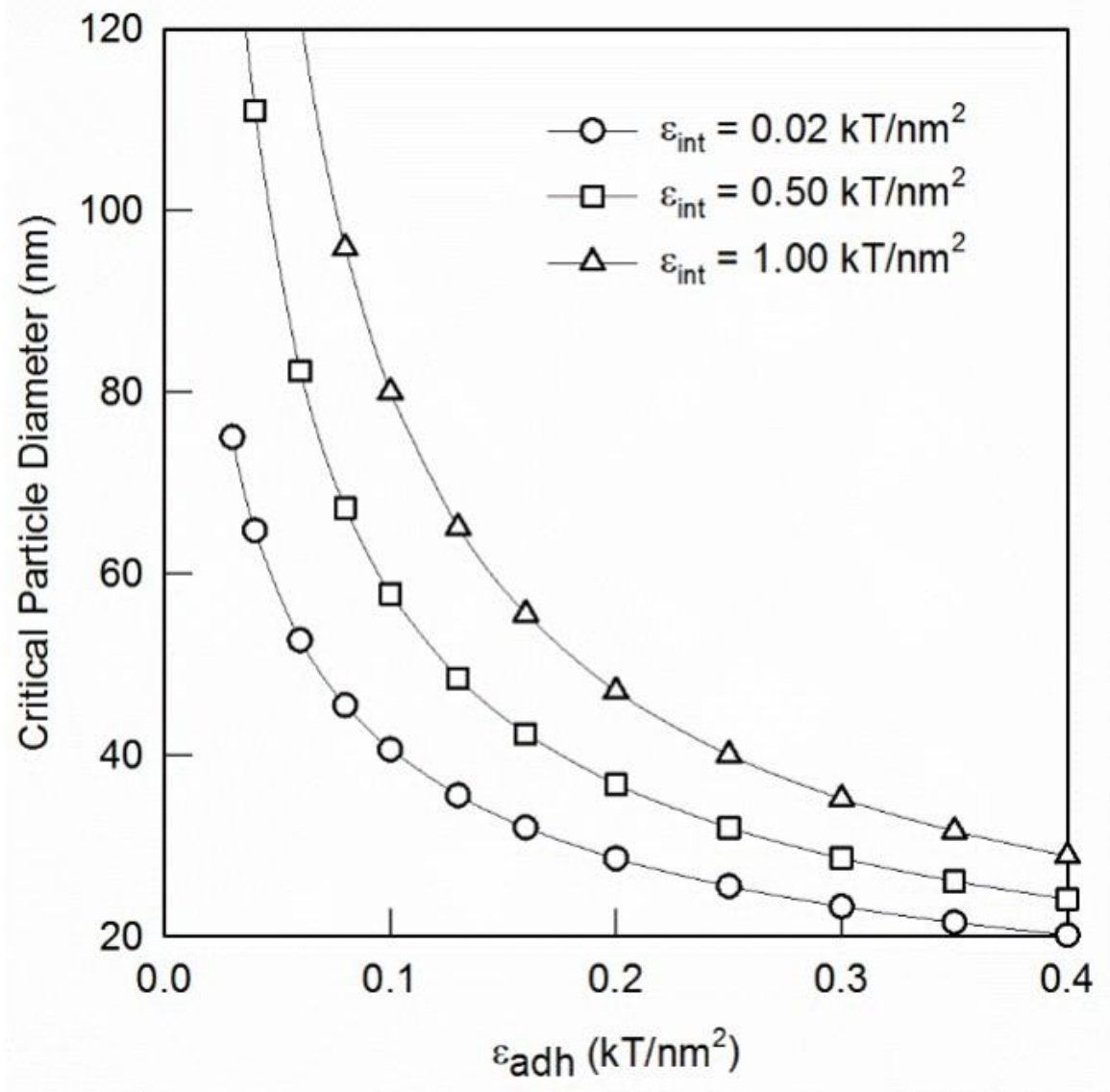

Figure 7 

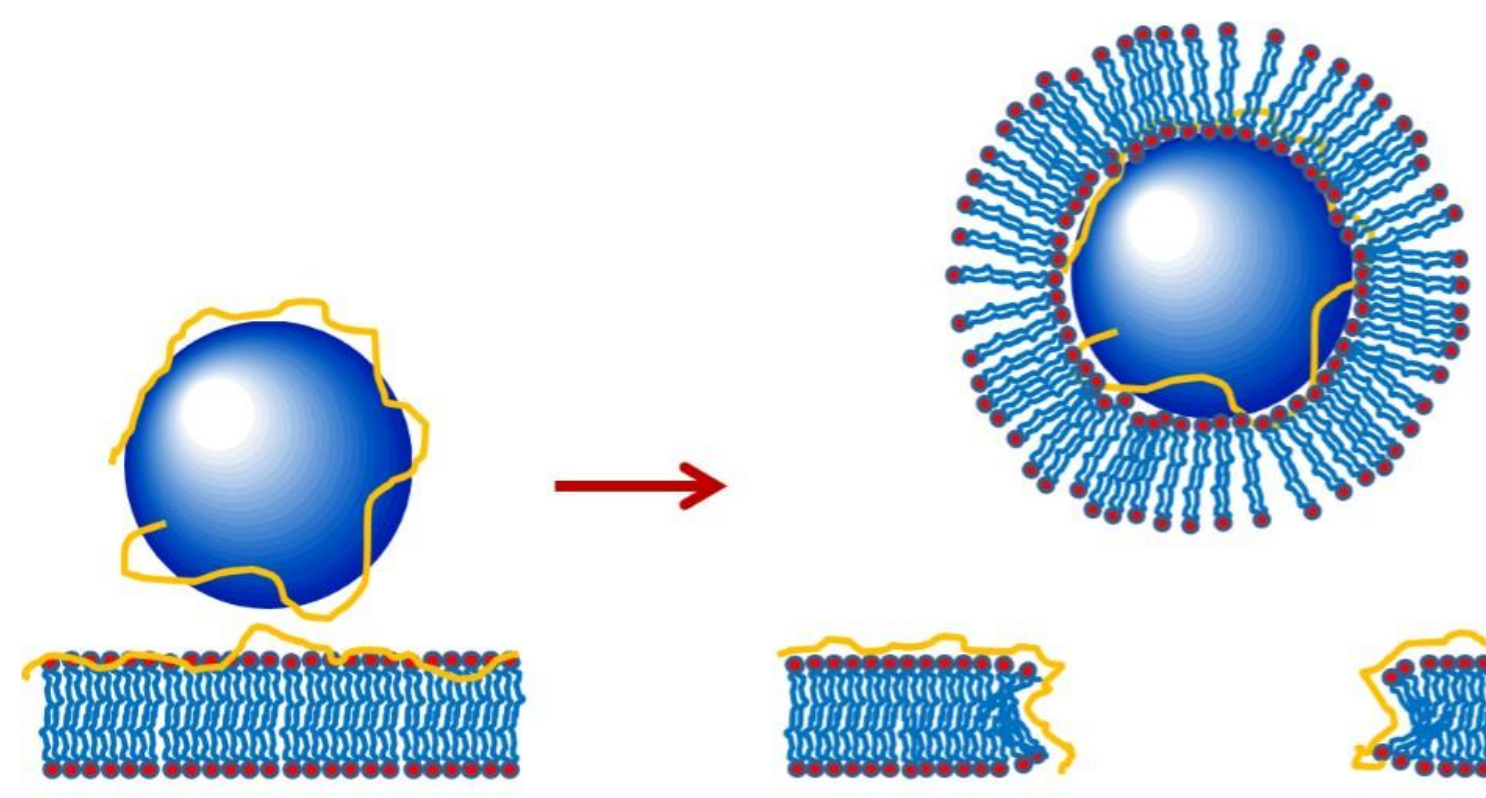

\section{$\Delta g=-4 \pi R_{P}^{2} \epsilon_{a d h}+8 \pi \kappa+4 \pi R_{P} \delta \epsilon_{\text {int }}$}

Graphical Abstract 